\title{
Editorial
}

\section{Recent Advances in Wireless Communication Protocols for Internet of Things}

\author{
Jiangchuan Liu, ${ }^{1}$ Feng Wang, ${ }^{2}$ Xiaoqiang $M a,{ }^{3}$ and Zhe Yang $^{4}$ \\ ${ }^{1}$ College of Natural Resources and Environment, South China Agricultural University, Guangzhou, Guangdong 510640, China \\ ${ }^{2}$ Department of Computer and Information Science, University of Mississippi, University, MS 38677-1848, USA \\ ${ }^{3}$ School of Electronic Information and Communications, Huazhong University of Science and Technology, Wuhan, \\ Hubei 430074, China \\ ${ }^{4}$ Northwestern Polytechnical University, Xian, Shaanxi 710065, China
}

Correspondence should be addressed to Jiangchuan Liu; jcliu@cs.sfu.ca

Received 18 October 2017; Accepted 18 October 2017; Published 16 November 2017

Copyright (C) 2017 Jiangchuan Liu et al. This is an open access article distributed under the Creative Commons Attribution License, which permits unrestricted use, distribution, and reproduction in any medium, provided the original work is properly cited.

Internet of Things (IoT) is one of the hottest research fields nowadays and has attracted huge interests and research efforts from both academia and industry. IoT can connect a large number of sensors, actuators, devices, vehicles, buildings, and/or other objects to form a network where data can be collected from the physical world, exchanged and processed in the cyber world, and then fed back into the physical world through actuations. This makes IoT one of the key foundations towards the vision of smart cities, with many promising applications such as environmental monitoring, infrastructure management, manufacturing, energy management, medical and healthcare, building and home automation, and transportation.

Recently, the advances in various wireless communication protocols in technologies such as 5G, RFID, Wi-FiDirect, Li-Fi, LTE, and 6LoWPAN have greatly boosted the potential capabilities of IoT and made it become more prevalent than ever, which also accelerate the further integration of IoT with emerging technologies in other areas such as sensing, wireless recharging, data exchanging, and processing. Yet, how these technologies especially the corresponding wireless communication protocols can be well aligned with IoT to maximize their benefits on such performance as scalability, service quality, energy efficiency, and cost effectiveness is still open to investigation and thus calls for novel solutions. And the involved privacy and security issues also need to be carefully examined and addressed.

This special issue aims to summarize the latest development in wireless communication protocols for Internet of
Things and how such development can enable other emerging technologies to be further integrated with IoT and boost its capabilities. We classify the accepted papers into four major focuses: (1) energy efficiency; (2) QoS awareness; (3) localization; and (4) security and privacy.

The energy efficiency focus consists of 2 papers.

The paper "Energy Harvesting for Internet of Things with Heterogeneous Users" studies the energy harvesting problem in the Internet of Things with heterogeneous users under multiuser MISO broadcast channels.

The paper "Profiling Energy Efficiency and Data Communications for Mobile Internet of Things" aims at monitoring the power consumption behaviors of the smartphones, profiling both individual applications and the entire system, to make better decisions in power management. The authors design a cloud orchestration architecture as an epic predictor of behaviors of smart devices by extracting their application characteristics and resource utilization.

The QoS awareness focus consists of 4 papers.

The paper "QoE-Driven D2D Media Services Distribution Scheme in Cellular Networks" proposes a novel media service scheme based on different QoE models that jointly solve the massive media content dissemination issue for cellular networks, where the authors investigate the socalled Media Service Adaptive Update Scheme (MSAUS) framework to maximize users' QoE satisfaction and derive the popularity and priority function of different media service QoE expression. A Media Service Resource Allocation (MSRA) algorithm is also designed to schedule limited 
cellular networks resource, which is based on the popularity function to optimize the total users' QoE satisfaction and avoid D2D interference.

The paper "Det-WiFi: A Multihop TDMA MAC Implementation for Industrial Deterministic Applications Based on Commodity 802.11 Hardware" proposes Det-WiFi, a realtime TDMA MAC implementation for high-speed multihop industrial application, which can support high-speed applications and provide deterministic network performance via combining the advantages of high-speed IEEE802.11 physical layer and a software Time Division Multiple Access (TDMA) based MAC layer.

As network coding is emerging as a promising technique that can provide significant improvements in the throughput of Internet of Things, the paper "Efficient Network Coding with Interference-Awareness and Neighbor States Updating in Wireless Networks" proposes a novel network coding scheme to achieve a higher throughput improvement with lower computational complexity and buffer occupancy than traditional greedy-based schemes, where topology knowledge is utilized to minimize interference and obtain more throughput, and a more reliable broadcast protocol is exploited to alleviate the decoding failure caused by the inherent error ratio in ETX.

The paper "Exploiting Delay Aware Load Balance for Scalable 802.11 PSM in Crowd Event Environments" presents ScaPSM (Scalable Power-Saving Mode scheduler), a design that enables scalable competing background traffic scheduling in crowd event 802.11 deployments with Power-Saving Mode (PSM) radio operation. The key novelty behind ScaPSM is that it exploits delay aware load balance to control judiciously the qualification and the number of competing PSM clients before every beacon frame's transmission, which helps to mitigate congestion at the peak period with increasing the number of PSM clients.

The localization focus consists of 3 papers.

As the Receiving Signal Strength (RSS) is the key foundation for IoT communication resource allocation, localization, interference management, sensing, and so forth, the paper "Compressed RSS Measurement for Communication and Sensing in the Internet of Things" proposes a compressive sensing-based RSS measurement solution, which takes advantage of compressive sensing theory to enable simultaneous measurement in the same channel, so as to achieve conflict-tolerant, time-efficient, and accuracyguaranteed without any model-calibrate operation.

The paper "Dealing with Insufficient Location Fingerprints in Wi-Fi Based Indoor Location Fingerprinting" targets one but common situation when the collected measurements on received signal strength information are insufficient and shows limitations of existing location fingerprinting methods in dealing with inadequate location fingerprints. The authors then introduce a novel method to reduce noise in measuring the received signal strength based on the maximum likelihood estimation and compute locations from inadequate location fingerprints by using the stochastic gradient descent algorithm.

The paper "SmartFix: Indoor Locating Optimization Algorithm for Energy-Constrained Wearable Devices" presents
SmartFix, an optimization algorithm for indoor locating based on Wi-Fi RSS, which utilizes user motion features and extracts characteristic value from history trajectory and corrects deviation caused by unstable Wi-Fi signals.

The security and privacy focus consists of 2 papers.

Given that covert communication is applied in many IoT scenarios for information transmission security, the paper "Collaborative Covert Communication Design Based on Lattice Reduction Aided Multiple User Detection Method" applies the lattice reduction theory to multiple access interference cancellation of spread spectrum communication and proposes a novel lattice reduction aided multiple user detection method to better design a covert communication system.

The paper "Privacy-Preserving Meter Report Protocol of Isolated Smart Grid Devices" proposes an efficient privacypreserving meter report protocol for the isolated smart grid devices, which consists of an encryption scheme with additively homomorphic property and a linearly homomorphic signature scheme suitable for privacy-preserving data aggregation.

\section{Acknowledgments}

We would like to thank all the reviewers who have participated in reviewing the articles submitted to this special issue.

$$
\begin{array}{r}
\text { Jiangchuan Liu } \\
\text { Feng Wang } \\
\text { Xiaoqiang Ma } \\
\text { Zhe Yang }
\end{array}
$$




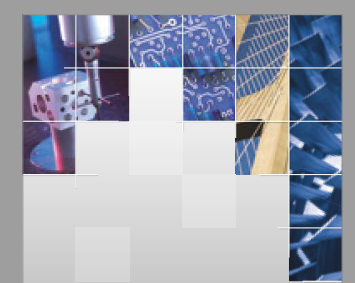

\section{Enfincering}
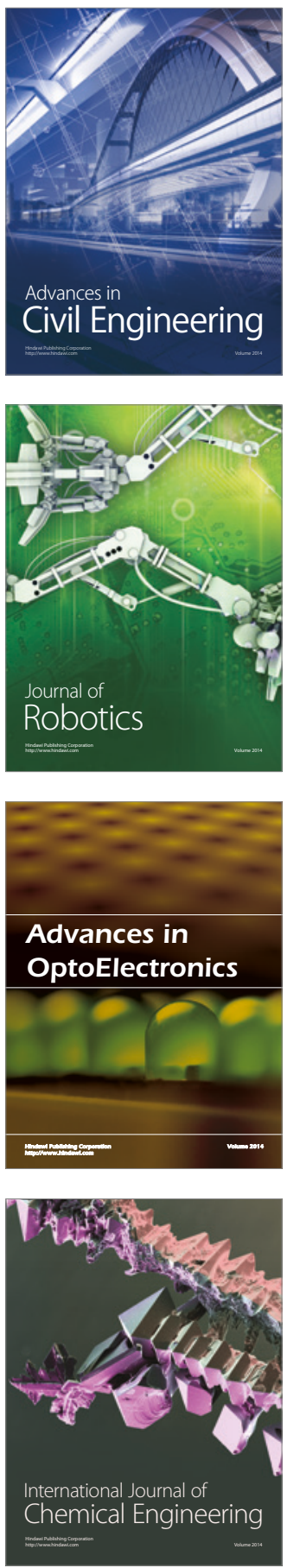

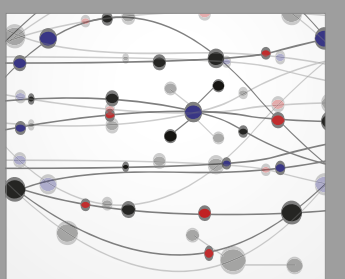

The Scientific World Journal

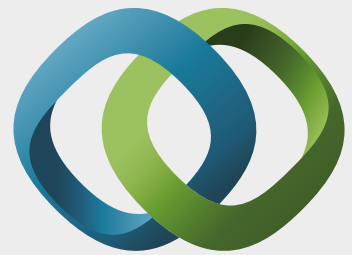

\section{Hindawi}

Submit your manuscripts at

https://www.hindawi.com
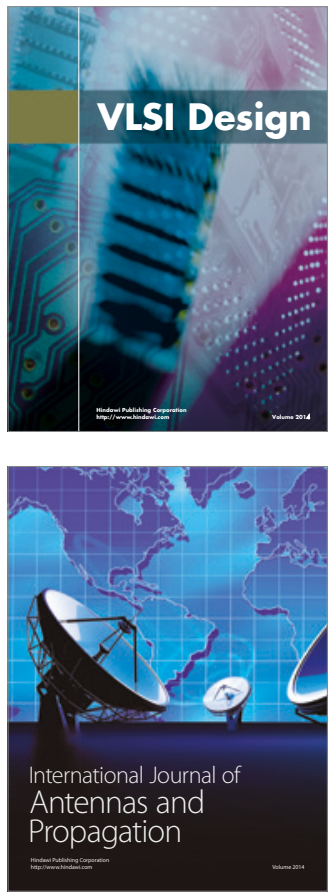

\section{Rotating}

Machinery
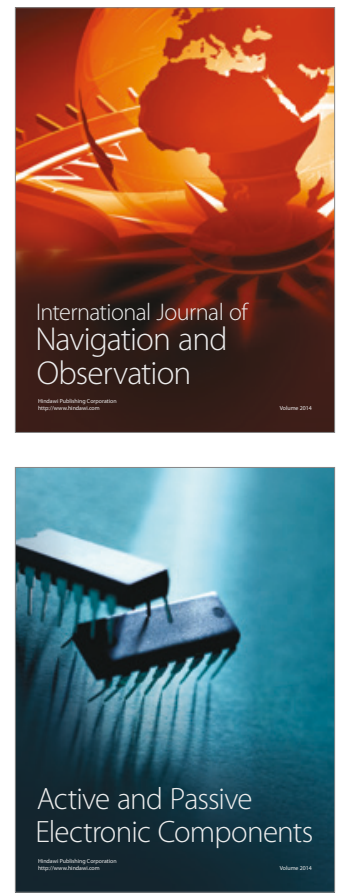
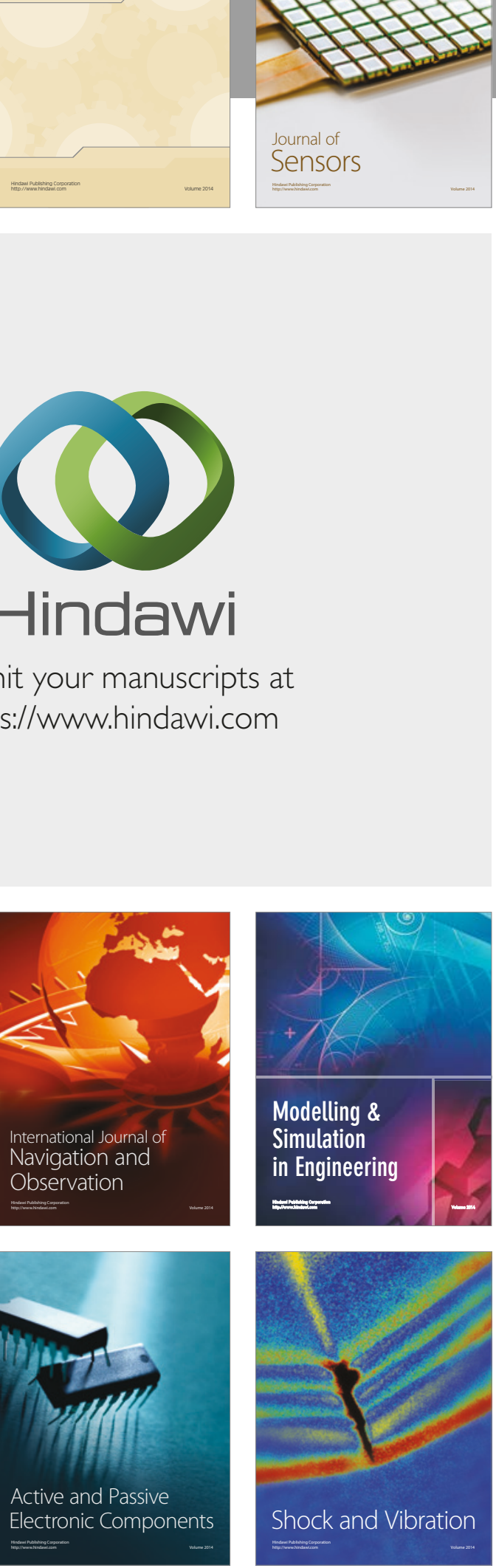
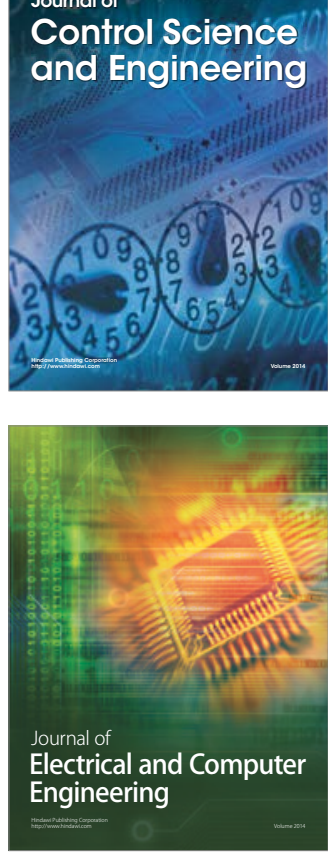

Distributed

Journal of

Control Science

and Engineering
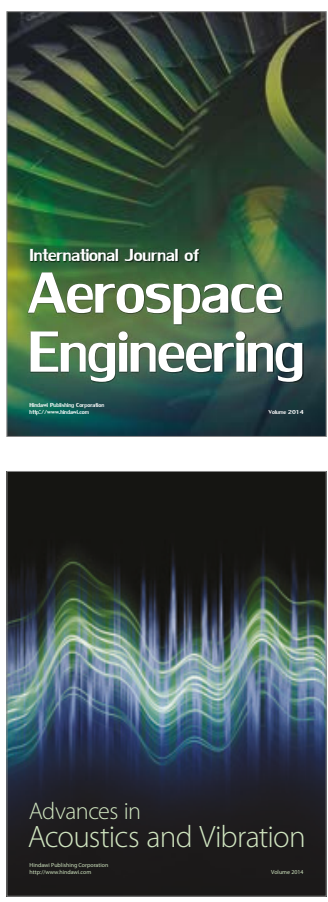

Sensor Networks 